\section{WEIRD languages have misled us, too}

\section{doi: $10.1017 /$ S0140525X1000018X}

\section{Asifa Majid and Stephen C. Levinson \\ Max Planck Institute for Psycholinguistics, Nijmegen 6500AH, The Netherlands. \\ asifa.majid@mpi.nl http://www.mpi.nl/people/majid-asifa stephen.levinson@mpi.nl \\ http://www.mpi.nl/people/levinson-stephen}

Abstract: The linguistic and cognitive sciences have severely underestimated the degree of linguistic diversity in the world. Part of the reason for this is that we have projected assumptions based on English and familiar languages onto the rest. We focus on some distortions this has introduced, especially in the study of semantics.

This commentary rounds out the picture convincingly advanced in the target paper by considering how scientific approaches to language have also ignored the diversity of behavior in the linguistic domain. In the BBS paper "The Myth of Language Universals," Evans and Levinson (2009) argue that there is little evidence for the view that the variation in language structure is tightly bounded by linguistic universals. Instead, what we find is extensive variation on almost every dimension, with the main patterns understandable in terms of cultural evolution.

Why does it matter to psychology that languages vary fundamentally on so many dimensions? Leaving aside that it is the highest learned human skill, and therefore has an interest in its own right, there are at least two further reasons: (a) Language is in many ways a "window on the mind," and (b) semantic variation seems to correlate with psychological variation on a range of parameters. As a result, most of our ideas about how humans reason or what notions form natural categories are prompted by our own languages.

We must leave to the historians of science an explanation for how the myth of language universals came to dominate the language sciences for 50 years. But one factor is almost certainly the view that familiar languages such as English are canonical. Yet many features of English are quite unusual - for example, only $1.6 \%$ of languages express Yes/No questions by word order inversion (Dryer 2008), and no other known language has verb inflection with non-zero exponent for third person (as in John come-s) but zero for all other persons (see rara\#34 http://typo.uni-konstanz.de/ rara/). As we discuss here, there are languages without such "natural" concepts as left, in, arm, or green. English is, in fact, in a special, rather unusual corner of the design space for human languages, an outlier, not some kind of central default model at all.

As surveyed in the target article (sect. 3.4), there appears to be significant variation in the spatial frames of reference employed crossculturally, largely predictable from language - that is, languages without spatial left and right terms are used by peoples who prefer allocentric coding. Some authors find these results hard to credit and have wondered whether they don't reflect conscious ratiocination correcting an innate egocentric bias ( Li \& Gleitman 2002). Haun and Rapold (2009) now produce additional evidence for the depth of allocentric coding in a non-WEIRD culture, Haillom hunter-gatherers of Namibia. Haun and Rapold asked 8year-olds to learn a demonstrated dance, and then got them to repeat the dance under 180 -degrees rotation. The Hai $\|$ om kids maintained the dance moves in absolute (North-South-EastWest) coordinates under rotation, whereas a matched German sample maintained the dance moves in egocentric coordinates (in terms of left/right motions). Given the rigidly egocentric nature of neural coding for body position, these findings are quite unexpected and show the extent to which a different spatial framework can be adopted in the cognitive representation of spatial movement.

The spatial encoding of topological relations between objects in non-WEIRD languages also challenges long-held assumptions about the human mind. Developmental psychologists and linguists have supposed that preverbal infants have a stock of prelinguistic concepts (either inborn or learned through sensorimotor experience) and that language maps onto these universal primitives (e.g., Clark 1973; Slobin 1973). These include notions such as in, on, and under - exactly those concepts found in English. Infants less than a year old show sophisticated understandings of such relations (Baillargeon 1994). But subsequent crosslinguistic investigation has shown considerable variation in how languages express spatial concepts (Bowerman 1996; Bowerman \& Choi 2001). Korean speakers distinguish between "tight-fitting" and "loose-fitting relations" instead of "containment" (as in in) or "support" (as in on). This finding has had a profound impact on how we currently think about the infant mind. Rather than the infant coming to the world with only English spatial categories, she comes now with Korean ones, too (Hespos \& Spelke 2002; McDonough et al. 2003), and researchers are actively pursuing which of the myriad further distinctions coded in other languages may be present for the infant, too.

In another domain, vision scientists have been impressed by the correspondence between the algorithms used by the visual system to parse the world around us and their reflection in language. Hoffman and Richards (1984, p. 82), after discussing how parts are assigned when viewing a face, conclude that: "It is probably no accident that the parts defined by minima are often easily assigned verbal labels" - the caveat being "in English." The parts assigned verbal labels in other languages differ substantially. In Jahai, a language spoken in the Malay Peninsula by a group of nomadic hunter-gatherers, speakers make fine-grained segmentations of the body and face: there is no term corresponding to face, arm, or leg (Burenhult 2006). Compare this with Lavukaleve, spoken by some thousand-odd subsistence fishers and farmers on the Solomon Islands. Lavukaleve speakers have a much coarser-grained system and use a single term to refer to arms and legs, with no finer-level conventionalized terms for the limbs (Terrill 2006). The correspondence between English part categories and those identified by the vision sciences has caused researchers to seriously misjudge the issues involved in a theory of the language-perception interface (Majid 2010).

Take color as yet another domain. Here language plays a critical role. Languages carve up the spectrum into a number of discrete categories, and it is these linguistic categories that are utilized in memory and perception (Davidoff et al. 1999; Gilbert et al. 2006). Speakers of languages from WEIRD societies make finer divisions in the color space than do speakers of most of the thousands of other languages of the world (Kay \& Maffi 2008), and the pinnacle of color categorization (in terms of how many divisions of the color space a language makes) has been taken to be 11 - exactly the number that English has (Berlin \& Kay 1969; Kay \& Maffi 2008). But there is accumulating evidence that WEIRD societies may be surpassed in this domain. In Russian (Corbett \& Davies 1995), Turkish (Özgen \& Davies 1998), Greek (Thierry et al. 2009), and Japanese (Uchikawa \& Boynton 1987), there are 12 terms (an additional distinction is made between dark and light blue). The new pinnacle is 15, as demonstrated by Korean (see Roberson et al. 2008).

Human performance diversity offers a rich resource for cognitive scientists. It allows us to triangulate on underlying properties of mind that would be invisible if we were all culturally identical. Instead of lamenting the loss of the "psychic unity of mankind," we should embrace the study of cognitive diversity as a window on human cognitive potential.

\section{WEIRD societies may be more compatible with human nature}

\section{doi:10.1017/S0140525X10000191}

\section{Alexandra Maryanski}

Department of Sociology, University of California-Riverside, Riverside, CA 92521.

alexandra.maryanski@ucr.edu

Abstract: Are WEIRD societies unrepresentative of humanity? According to Henrich et al, they are not useful for generalizing about 